\title{
Chemostat culture systems support diverse bacteriophage communities from human feces
}

Tasha M. Santiago-Rodriguez ${ }^{1}$, Melissa Ly' ${ }^{1}$ Michelle C. Daigneault², lan H. L. Brown², Julie A. K. McDonald², Natasha Bonilla ${ }^{3}$ Emma Allen Vercoe ${ }^{2}$ and David T. Pride ${ }^{1,4^{*}}$

\begin{abstract}
Background: Most human microbiota studies focus on bacteria inhabiting body surfaces, but these surfaces also are home to large populations of viruses. Many are bacteriophages, and their role in driving bacterial diversity is difficult to decipher without the use of in vitro ecosystems that can reproduce human microbial communities.

Results: We used chemostat culture systems known to harbor diverse fecal bacteria to decipher whether these cultures also are home to phage communities. We found that there are vast viral communities inhabiting these ecosystems, with estimated concentrations similar to those found in human feces. The viral communities are composed entirely of bacteriophages and likely contain both temperate and lytic phages based on their similarities to other known phages. We examined the cultured phage communities at five separate time points over 24 days and found that they were highly individual-specific, suggesting that much of the subject-specificity found in human viromes also is captured by this culture-based system. A high proportion of the community membership is conserved over time, but the cultured communities maintain more similarity with other intra-subject cultures than they do to human feces. In four of the five subjects, estimated viral diversity between fecal and cultured communities was highly similar.
\end{abstract}

Conclusions: Because the diversity of phages in these cultured fecal communities have similarities to those found in humans, we believe these communities can serve as valuable ecosystems to help uncover the role of phages in human microbial communities.

Keywords: Stool, Feces, Bacteriophage, Microbiome, Virome, Metagenome, Chemostat, Viral communities

\section{Background}

Human body surfaces are inhabited by diverse viral communities, and the majority of those identifiable viruses are bacteriophages $[1,2]$. The most well studied viral communities to date are those in the human gut and the oral cavity. Viruses in the gut are rapidly evolving [3], exhibiting subject-specificity [4], responding to dietary changes, and persisting over time [5]. Similarly, viruses in the oral cavity are highly personalized [6] and highly persistent, possibly as a result of their ability to evade defense mechanisms utilized by oral bacteria [6-8]. The oral cavity has different biogeographic sites with specific

\footnotetext{
* Correspondence: dpride@ucsd.edu

'Department of Pathology, University of California, 9500 Gilman Drive, MC 0612, La Jolla, CA 92093-0612, USA

${ }^{4}$ Department of Medicine, University of California, San Diego, CA, USA Full list of author information is available at the end of the article
}

viral communities whose membership differs significantly in periodontal health and disease [9]. Limitations due to virus assembly from metagenome data, however, often result in overestimations of the diversity present in human viral communities [10]. There also are viral communities inhabiting human skin [11] and the respiratory tract [12], but the role of viruses as members of the human microbial communities is not well understood.

Bacteriophages possess the capacity to alter microbial communities by either lysing their hosts or providing phenotypic advantages to recipient bacteria [13-15]. Phages may influence biogeochemical cycles in aquatic environments by decreasing the relative abundances of specific bacterial species $[16,17]$ or by supporting bacterial populations with the wide repertoire of metabolicassociated genes they possess $[18,19]$. The ability of 
phages to influence microbial diversity has been hypothesized to have consequences for human health by altering the normal microbiota that may have protective effects against colonization by more pathogenic microorganisms. While some phages have lysogenic lifestyles and may contribute gene functions to their hosts in human microbial communities, others have lytic lifestyles and may be responsible for driving microbial diversity on human body surfaces. The subgingival crevice in periodontal disease is enriched for myoviruses [9] that typically have lytic lifestyles, which implicates them as potential drivers of microbial diversity in human disease. That microbial diversity in human disease that may be potentiated by viruses provides a significant rationale for more studies focused on the role of viruses as members of human microbial communities.

Studies of microbiota can be limited due to restrictions imposed by working with human subjects [20]. For example, examining the effects of a lytic virus on human microbiota meets with ethical challenges that limit experimental design. Some studies have been valuable in discerning the effects of perturbations on human gut microbiota, but personalized individual microbial profiles and individual confounders such as age, diet, and medications may influence gut microbial composition [21-26]. The restrictions associated with working with human subjects and the potential for significant confounders despite well-designed experiments necessitate the development of cultivation-based systems capable of reproducing the complex interactions of the microbiota on human body surfaces. There currently are no known cultivation-based ecosystems that effectively reproduce the diversity of viral populations in humans.

Model microbial systems must be reproducible, highly stable, and must retain similar levels of diversity to the inocula from which they were derived [27, 28]. Interactions between host and phage have been previously modeled in gnotobiotic mice [29], which allowed for the tracking of phage dynamics with their individual hosts. The cellular microbiota of the human gut has been modeled by cultivating fecal samples in complex chemostat systems [20,30]. These microbial communities reach an equilibrium that resembles the gut community structure from which they originated [27, 31-33]. Models of the oral microbiota in other culture-based systems also have been shown to reproduce much of the taxonomical and functional characteristics of the oral biofilm [34]. These systems offer the potential to study the response of microorganisms to perturbations in controllable and reproducible environments that reduce the potential for confounders that often are encountered working with human subjects [20,35]. None of these cultivation-based ecosystems have been shown to be inhabited by viral communities, but researchers previously have successfully cultivated individual viruses in these types of systems [36, 37]. We believe that because chemostat culture systems effectively reproduce much of the cellular microbiota in the gut, they may also be home to substantial viral communities. The goals of this study were as followings: (1) to demonstrate that cultured fecal microbial communities are home to robust viral communities, (2) to develop techniques to investigate the diversity of the viral communities in cultured fecal communities, and (3) to determine whether cultured fecal communities have similarities to viral communities present in human feces.

\section{Results}

\section{Human subjects and chemostat cultured communities}

We recruited five human subjects through the University of Guelph and sampled their feces. Donors \#1, \#2, and \#10 were a co-habiting family unit of father, mother, and child, respectively, while donors \#8 and \#9 were unrelated. Each fecal sample was homogenized and processed immediately into a chemostat vessel, and the remainder of the feces was stored until processing of the viromes could take place. Chemostat vessels were operated under conditions designed to mimic the human distal colon [30]. Cultured microbial communities were taken from donors \#1, \#2, and \#10 on days $4,8,12,16$, and 24 and from donors $\# 8$ and $\# 9$ on days $3,6,12,18$, and 24 . Previous studies have shown that cellular microbial communities reach stable state in culture by day 24 [30]. We tested for the presence of fluorescent-staining particles (FSPs) in feces and cultures, similar to those previously described, to indicate the likely presence of viruses in both sample types [38]. On day 24, we found that there were numerous FSPs in both sample types, with a mean $3.7 \times 10^{9}$ FSPs in feces and $1.4 \times 10^{9}$ FSPs in the chemostat cultures for all subjects studied. The presence of such high densities of FSPs in both sample types strongly suggested the presence of substantial viral communities.

We isolated and processed viruses from both feces and chemostat cultures utilizing sequential filtration followed by cesium chloride density gradient centrifugation according to our previously described protocols [39]. We sequenced the resulting viral DNA from the feces and chemostat cultures of the five donors using semiconductor sequencing [40] and produced 18,584,604 reads $(619,487$ reads per time point and sample type) of mean length 215 nucleotides (Additional file 1: Table S1). We used BLASTN to compare all viromes to the RDP $16 \mathrm{~S}$ rRNA genes database (E-value $<10^{-5}$ ) and found that all were free of 16S rRNA gene homologues. We also used BLASTN to search the viromes for homologues against a human reference genome, and some similar sequences (E-value $<10^{-5}$ ) were identified and removed prior to further analysis. These data suggest that these chemostat and fecal viromes were relatively free of contaminating cellular nucleic acids. 


\section{Identification of viruses and viral families in cultured fecal material}

We assembled virome reads from each subject to construct longer contigs, as this generally results in more productive searches for sequence similarities. We used several different assemblers including CLC Genomics Workbench, MetaVelvet [41], and IDBA-UD [42] in the construction of contigs and found that CLC Genomics Workbench produced fewer contigs with longer mean and maximum lengths and higher N50 values than the other assemblers tested (Additional file 2: Table S2). With the CLC assembler, $96.4 \pm 1.3 \%$ of all reads were assembled into contigs. Therefore, we utilized the contigs constructed using CLC Genomics Workbench throughout the study. The mean GC content for all contigs was $45.5 \%$ (mean of $46.3 \%$ for cultured viromes and $41.7 \%$ for fecal viromes; $p=0.01$ ) (Additional file 3: Figure S1, Panel A). The mean length amongst all contigs was 941 nucleotides (mean of 908 nucleotides for cultured viromes and 965 nucleotides for fecal viromes; $p=0.35$ ) (Additional file 3: Figure S1, Panel B). The differences identified in GC content suggest that there may be features of viromes that are specific to both fecal and cultured communities.

Prior studies of viromes generally have identified large proportions of putative viruses that have no significant sequence similarities in available databases. We used BLASTX analysis of the assembled contigs against the NCBI non-redundant database (NR) to determine the relative proportion of our viromes that had significant sequence similarities. We utilized the numbers of reads assigned to each contig to identify the proportions of reads belonging to contigs with significant sequence similarities in the NR database. We found that in all subjects combined, $94.9 \pm 1.7 \%$ of the reads belonged to contigs that had similar sequences in the NR database (Fig. 1); $88.0 \pm 10.2 \%$ of those reads belonged to contigs that had sequence similarities to phages, $6.9 \pm 9.9 \%$ belonged to contigs that were similar to bacteria, and $5.1 \pm 1.7 \%$ belonged to contigs with no significant sequence similarities in the NR database. Most virome studies have contigs that have significant sequence similarities to bacteria; however, in the absence of finding $16 \mathrm{~S}$ rRNA genes in any of the viromes, the similarities to bacteria likely represent annotation deficiencies rather than bacterial contamination. These data strongly indicate that by analysis of assembled contigs, we gain a more comprehensive view of the constituents of viromes than is typically observed from analysis of virome reads.

To decipher whether there may be viruses present in the cultured viral communities similar to those present in other public databases, we mapped the reads from each subject against a composite database of known viruses including the NCBI viral database and the Phantome database. We found that there were numerous different viruses that were matched by reads from the cultured viral communities. For example, many reads from day 24 mapped to Enterobacteria Phage FIAA91ss, including 1280 reads $(0.14 \%$ of the reads) from donor \#1 (Fig. 2a) and 1981 reads (0.25\% of the reads) from donor \#2 (Fig. 2b). Very few reads matching Phage FIAA91ss were found in donor \#1 on days 4, 8, 12, and 16 (Additional file 3: Figure S2, Panel A). Similar

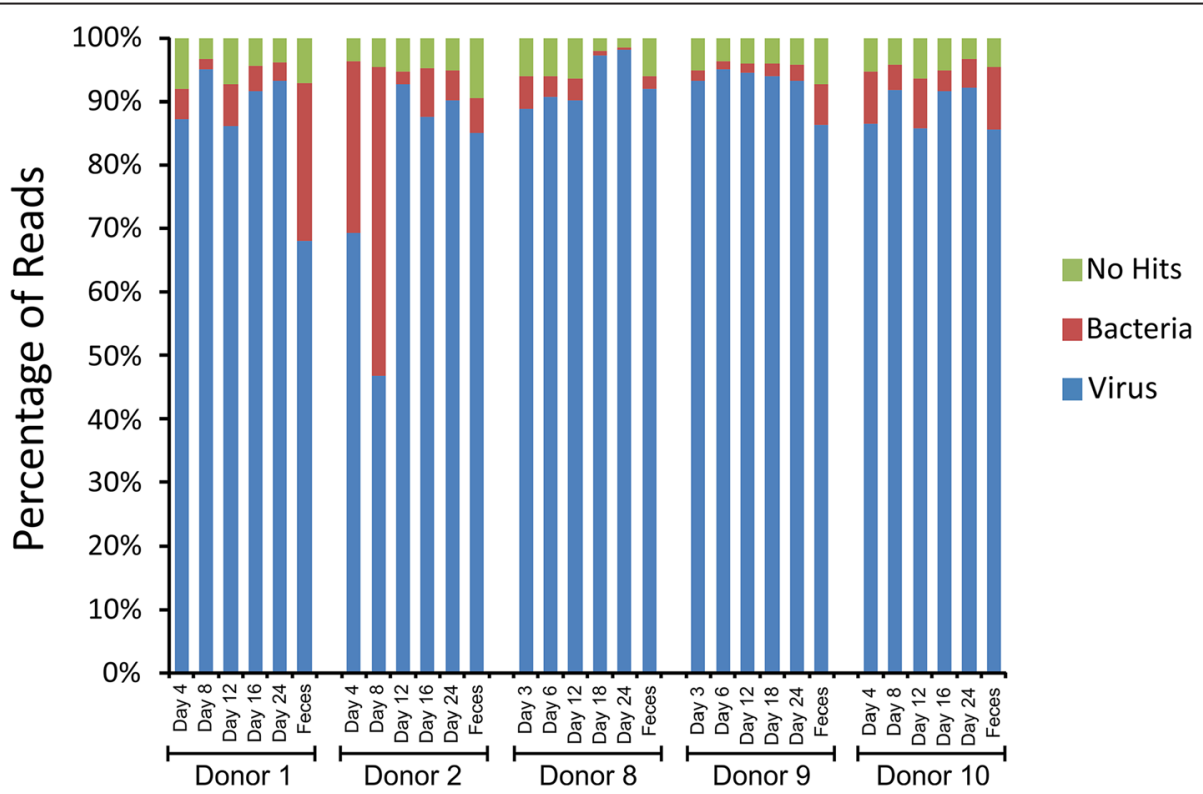

Fig. 1 Plots of percentage of virome reads that belong to contigs with significant sequence similarities in the NCBI NR database. The percentage of reads is demonstrated on the $\mathrm{Y}$-axis, and each donor, time point, and sample type is demonstrated on the $\mathrm{X}$-axis 


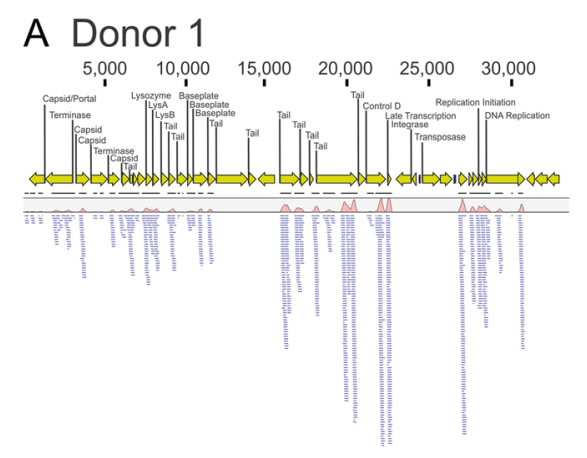

C Donor 1

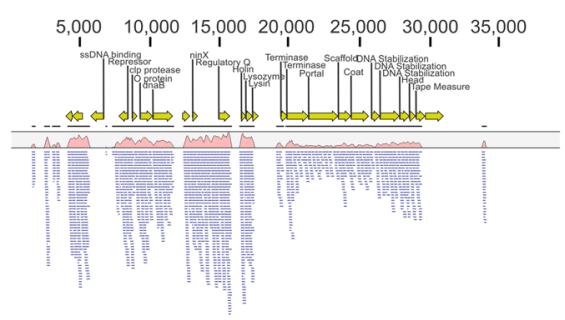

B Donor 2

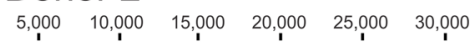

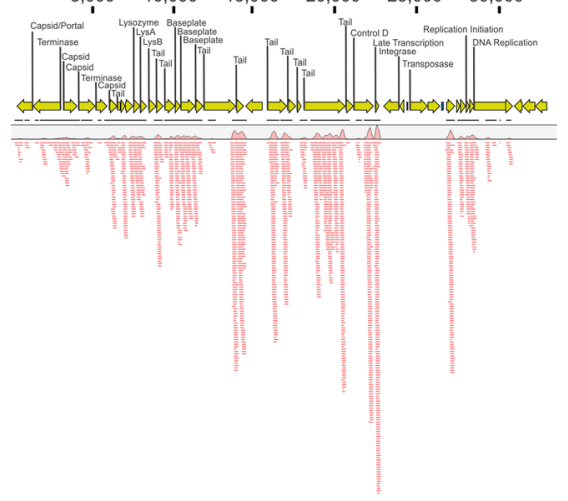

D Donor 2

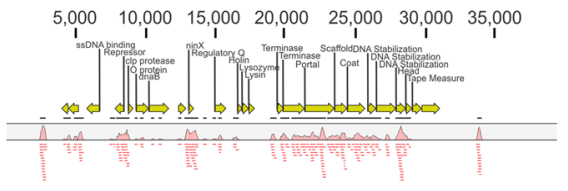

Fig. 2 Read mappings of chemostat viromes from day 24 to Enterobacteria phage FIAA91ss (a and b) and Enterobacteria phage IME10 (c and d). $\mathbf{a}$ and $\mathbf{c}$ represent donor \#1 and $\mathbf{b}$ and $\mathbf{d}$ represent donor \#2. The genes and their respective directions are shown by the yellow arrows, and the annotation of each gene is represented above. The relative location along the phage genomes are demonstrated by the scale at the top of the diagram, and the relative proportion of reads mapping to the phages are shown at the lower portion of the diagrams

results were found for donor \#2; however, a higher number of reads were identified on day 12 and their proportion increased up to day 24 (Additional file 3: Figure S2, Panel B). Enterobacteria Phage FIAA91ss is a myovirus, and although many myoviruses lead primarily lytic lifestyles, the presence of predicted integrase and transposase genes in the FIAA91ss genome suggests that like other P2-like phages, it has a primarily lysogenic lifestyle. There also were many reads from day 24 that mapped specifically to Enterobacteria Phage IME10, including 985 reads $(0.11 \%$ of the reads) from donor \#1 (Fig. 2c) and 255 reads (0.03\% of the reads) from donor \#2 (Fig. 2d). Unlike Phage FIAA91ss, Phage IME10 was found in both donors at all time points (Additional file 3: Figure S3). Enterobacteria Phage IME10 is a podovirus, a group which generally lead lytic lifestyles; however, Phage IME10 has a predicted repressor protein that suggests it may be lysogenic. Many reads from both donors also mapped to Enterobacteria Phage HK620 at all time points, suggesting that a similar phage is present in the cultured viromes of both donors (Additional file 3: Figure S4). Interestingly, reads matching Enterobacteria phages FIAA91ss, IME10, and HK620 were identified in donors \#1 and \#2 (husband and wife) but were not identified in donors \#10 (daughter), \#8, or \#9.

We also analyzed the cultured viral communities using the MG-RAST Server [43] and categorized those with sequences similar to known phages according to their families. We found that in all five donors, their cultured communities at all time points had reads similar to known myoviruses, podoviruses, and siphoviruses (Additional file 3: Figure S5). The proportions of reads similar to those different phage families generally differed substantially within a subject over time and between different subjects. The relative proportions of phage families observed do not necessarily reflect those found in the fecal viromes of each subject, particularly in donors \#8 and \#9. Viruses from the family Microviridae also were found in the feces of four of the five subjects, but none were identified in the chemostat cultures.

\section{Comparisons of fecal and cultured viral communities}

We identified significant sequence similarities to each assembled viral contig by BLASTX analysis against the NCBI non-redundant database to decipher which viral genes had similarities in the chemostat cultures. The vast majority of the contigs had similarities to hypothetical phage proteins, proteins involved in replication/integration, restriction/modification enzymes, or tail fibers (Additional file 3: Figure S6). There were no significant differences identified in the relative proportions of contigs similar to different phage categories for either fecal 
or cultured phage communities regardless of the time point examined.

We previously demonstrated that phages in the mouth are highly persistent members of human oral microbial communities [10]. We utilized similar techniques to decipher whether phages in the chemostat cultures might also persist over time. By creating viral assemblies from all time points within a donor combined, we then could assess which different time points contributed to each assembly. We found that $45 \pm 15 \%$ of assemblies from all subjects included contigs from day $4,42 \pm 8 \%$ from day $8,44 \pm 10 \%$ from day $12,38 \pm 17 \%$ from day $16,37 \pm 18 \%$ from day 24 , and $19 \pm 7 \%$ from feces (Additional file 3: Figures S7 and S8). The substantial difference in the percentage of assemblies that included fecal contigs suggests that there is less conservation in the phages present in feces compared to chemostat cultures. We also used BLASTN to compare the contigs between all donors and time points studied and found a similar pattern of conserved viruses over time in the chemostat cultures (Additional file 3: Figure S9). There was generally less conservation when comparing the chemostat culture viromes with those of the feces. The patterns of similar viruses across all donors suggested that there were individual-specific features of the viromes in each donor, giving the heatmap a "matrix-like" appearance. There was considerable similarity amongst the chemostat and fecal viromes of donors \#2 and \#10 (mother and child). To verify that the patterns of shared viruses we observed in the heatmap (Additional file 3: Figure S9) and the assemblies (Additional file 3: Figure S7) were statistically significant, we utilized a permutation test [44] to compare the proportions of shared viruses by sample type. For the cultured communities, $25 \%$ of the virus contigs sampled had significant sequence similarities across all subjects compared with only $9 \%$ when comparing cultured communities with fecal communities (Table 1), a difference that was close to statistical significance $(p=0.057)$. Similar results were found for fecal communities, where $25 \%$ of the viruses sampled across subjects had significant sequence similarities compared with only $13 \%$ when comparing between cultured communities and fecal communities $(p=0.141)$.

We created assemblies from all contigs in each donor to determine the relative proportions of viral contigs that were conserved in each donor over time. In donor \#1,
$53.3 \pm 4.0 \%$ of the contigs were conserved amongst the chemostat cultures over time compared to $25.9 \pm 3.0 \%$ conserved between the chemostat cultures and the feces (Fig. 3). In donor \#2, 62.3 $\pm 6.2 \%$ were conserved in chemostats compared to $26.5 \pm 4.2 \%$ between feces and chemostats; in donor \#8, $44.3 \pm 17.5 \%$ compared to $18.6 \pm 4.4 \%$; in donor \#9, $45.4 \pm 5.5 \%$ compared to $32.2 \pm 11.6 \%$; and in donor \#10, $65.3 \pm 7.1 \%$ compared to $18.9 \pm 3.5 \%$. In the chemostat cultures, the greatest conservation generally was between days 16 and 24, with $64.6 \pm 8.4 \%$ of the viral contigs conserved. These data indicate that while there are shared viruses between the chemostat cultures and the feces, there are identifiable differences in viral ecology between the sample types. Because our data also suggested that there were individual-specific features of each cultured virome, we used a permutation test to verify that the viral communities were significantly individual-specific across all time points in the cultured communities (Table 2). In all subjects studied, there was a statistically significant $(p \leq 0.05)$ trend observed, where the viruses in each of the cultured communities were significantly individual-specific.

We identified thousands of assemblies from all donors constructed from many different time points (Additional file 3: Figures S7 and S8). Each of these assemblies had identifiable phage sequence similarities. For example, in donor \#9, we identified a 36,261 bp contig with numerous sequence similarities to phage genes across its length including hydrolase, helicase, and tape measure genes (Fig. 4). Similar results could be found for all donors; however, not all time points contributed equally to each assembly (Additional file 3: Figures S10, S11, S12 and S13). Interestingly, many of the assembled viruses had identifiable restriction/modification genes, which corroborate our findings of a high number of contigs with significant similarities to restriction/modification enzymes in the chemostat viromes (Additional file 3: Figure S6). M23 peptidases (Additional file 3: Figure S10), toxinantitoxin genes (Additional file 3: Figure S11), S-layer, and platelet-binding proteins (Additional file 3: Figure S12) all had similar sequences identified in the phage genomes. A phage from donor \#8 shared some synteny with crAssphage [45] (Additional file 3: Figure S13).

We utilized the taxonomic information from the virome BLASTX hits to determine whether the phages from fecal and cultured communities had similar profiles. We found

Table 1 Viral homologues within and between subject groups

\begin{tabular}{llll}
\hline & \multicolumn{2}{l}{ Virome } & \\
\cline { 2 - 4 } & Percent similar within group ${ }^{\mathrm{a}}$ & Percent similar between groups $^{\mathrm{a}}$ & \\
\hline Feces & $25.04 \pm 12.81$ & $8.69 \pm 5.44$ & 0.0568 \\
Chemostat & $25.41 \pm 10.23$ & $13.15 \pm 8.11$ & 0.1410 \\
\hline
\end{tabular}

${ }^{\mathrm{a}}$ Based on the mean of 10,000 iterations. One thousand random contigs were sampled per iteration

${ }^{\mathrm{b}}$ Empirical $p$ value based on the fraction of times the estimated percent similar contigs for each group exceeded that between groups 


\section{A Donor 1}

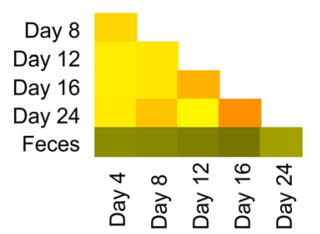

\section{Donor 9}

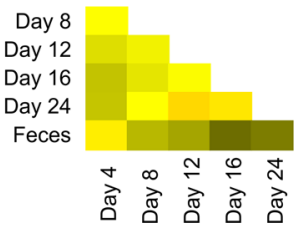

B Donor 2

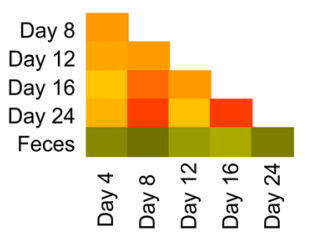

\section{E Donor 10}

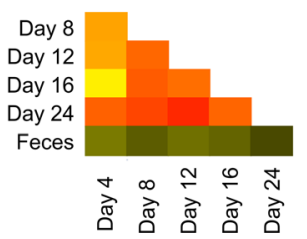

C Donor 8
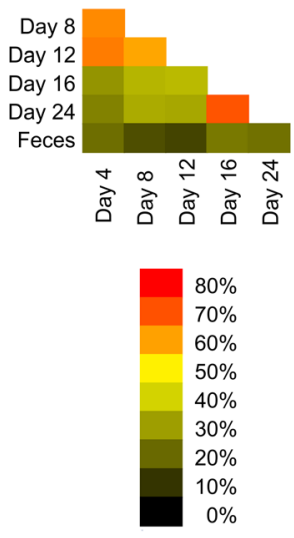

Fig. 3 Heat matrices of the percentage of assemblies from each donor (a-e) that contained contigs from each time point and sample type

that the profiles of BLASTX hits differed between the different donors and varied based on the time point examined (Fig. 5). Within each donor, the profiles were somewhat similar over time with the most substantial profile differences between the chemostat cultures and the feces in most subjects. The most abundant phyla identified were Bacteroidetes and Proteobacteria, but Firmicutes, Fusobacteria, and Verrucomicrobia also were identified. There was a relatively high number of Verrucomicrobia identified in donors 8 and 10, which represented the genus Akkermansia. For comparison, we characterized the taxonomy of the bacterial communities in fecal and cultured communities using $16 \mathrm{~S}$ rRNA genes. As expected, operational taxonomic units (OTUs) belonging to phyla Bacteroidetes and Firmicutes were amongst the most highly abundant taxa identified (Additional file 3: Figure S14).

We utilized principal coordinates analysis (PCOA) based on the beta diversity between the fecal and cultured viromes to determine if there were patterns of variation specific to each subject and sample type. Each of the cultured viromes reflected the subject from which they were derived (Fig. 6a). While the variation present in fecal viromes could be distinguished from the cultured viromes, they clustered near the cultured viromes in each donor, indicating that there were shared features between the fecal and cultured viromes. The patterns of variation observed on PCOA were highly robust, as similar patterns were observed when the PCOAs were constructed based on contigs contributing to assemblies (Additional file 3: Figure S15A) and BLASTX hit profiles (Figure S15B). The distinction between the different donors was not as apparent when examining the bacteria using 16S rRNA genes (Fig. 6b). These data indicate that much of the individualspecific character of human fecal viromes was captured in chemostat culture systems.

\section{Viral diversity in fecal and cultured communities}

We developed tools for characterizing viral communities to discern whether richness and diversity were conserved between fecal and cultured communities. The technique, termed the Homologous Virus Diversity Index (HVDI), is primarily based upon the Shannon Index [46] and is used for comparing diversity between different communities. The HVDI utilizes contig spectra as a surrogate for population structures and corrects for the limitations imposed on the contig spectra by assembly methods by assigning the

Table 2 Chemostat and fecal virome homologues within and between subjects

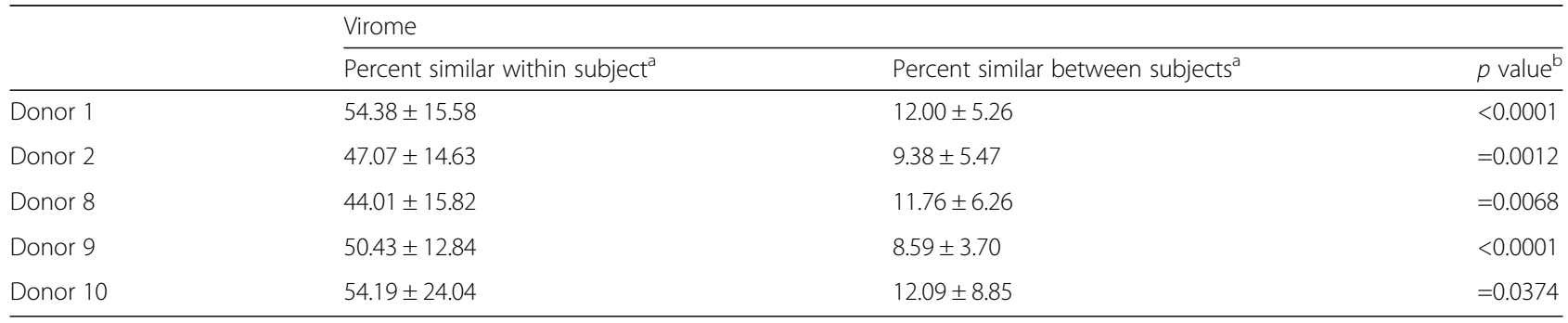

${ }^{a}$ Based on the mean of 10,000 iterations. One thousand random contigs were sampled per iteration

${ }^{\mathrm{b}}$ Empirical $p$ value based on the fraction of times the estimated percent similar contigs for each group exceeded that between group 


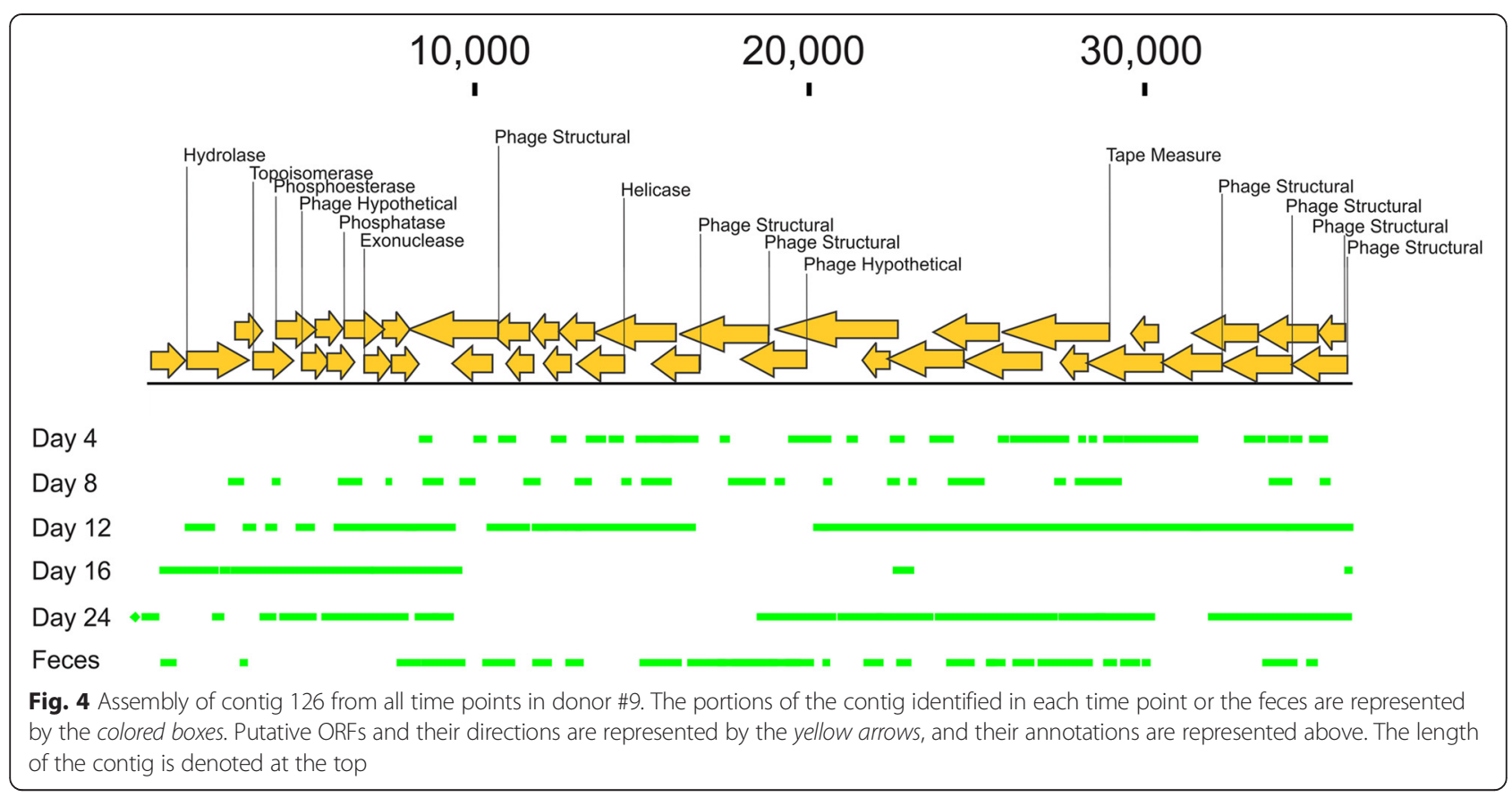

spectra for highly similar contigs to the same viral genotypes [47]. We validated the technique by randomly constructing viromes using viruses from the NCBI virus and Phantome databases to meet specific genotype and evenness requirements. Viromes were constructed with 10, 50, 100,500 , and 1000 different viral genotypes across an evenness spectrum consisting of $0.10,0.33,0.50,0.67$, and 0.90 . We created 10 separate viromes at each genotype and evenness value to ensure the data were reproducible and used the number of genotypes and randomly sampled reads from each virome to calculate the Shannon Index (Fig. 7). We then used the contig spectra from the assembled reads from each virome as input for the HVDI. When only 10 different viruses were evaluated, the HVDI values approximate the Shannon Index across all evenness levels (Fig. 7a), and similar results were found for 50 viral genotypes (Fig. 7b). At higher numbers of genotypes (100, 500, and 1000), the HVDI exceeded the Shannon Index, although not considerably (Fig. 7c-e). The extent of the diversity overestimates were related to the evenness in the viromes,

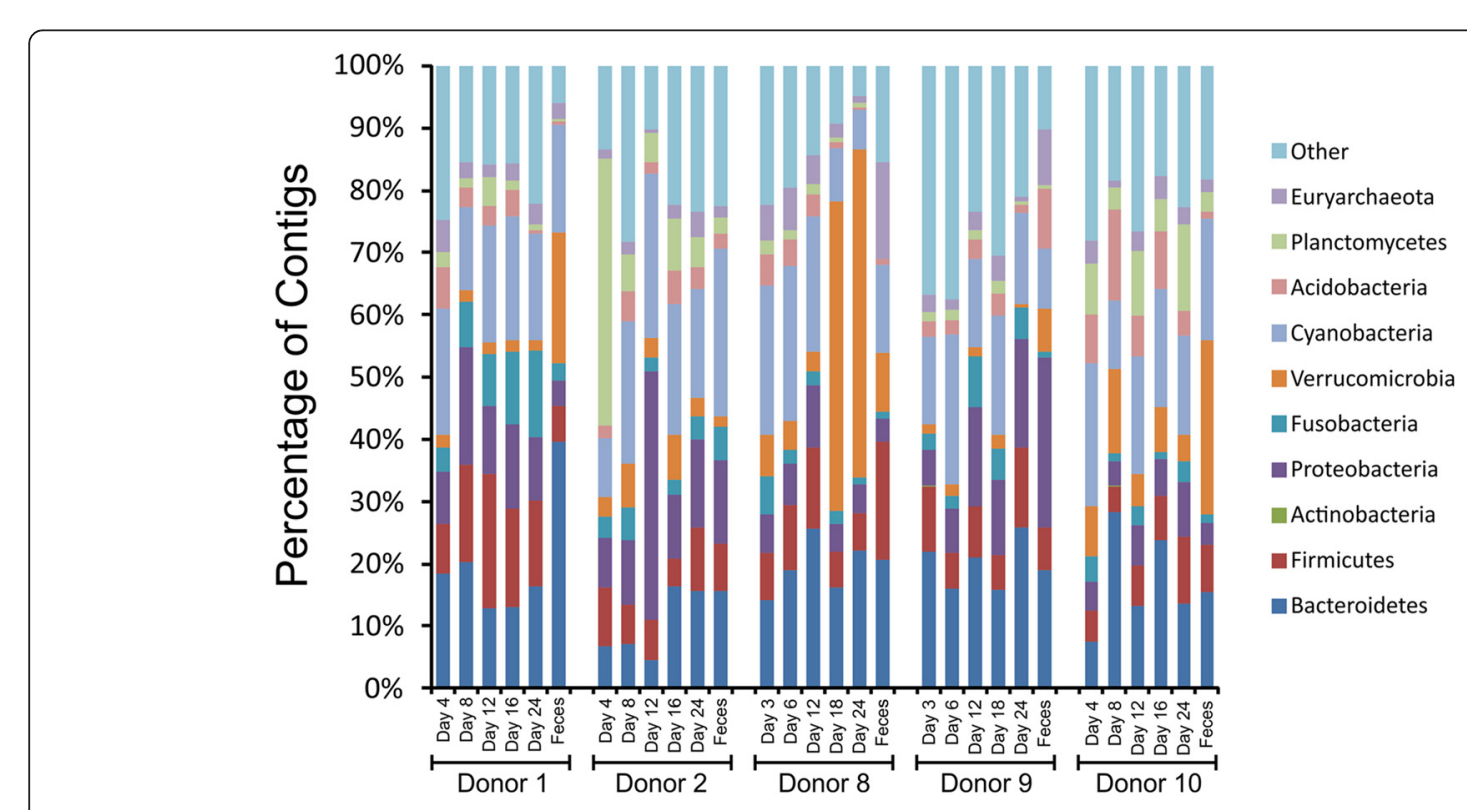

Fig. 5 Bar graphs demonstrating proportion of contigs with BLASTX significant sequence similarities to phages that parasitize the specified bacterial phyla for fecal and chemostat viromes. Each bar from left to right represents the day of culture, and the last bar for each donor represents fecal viromes 

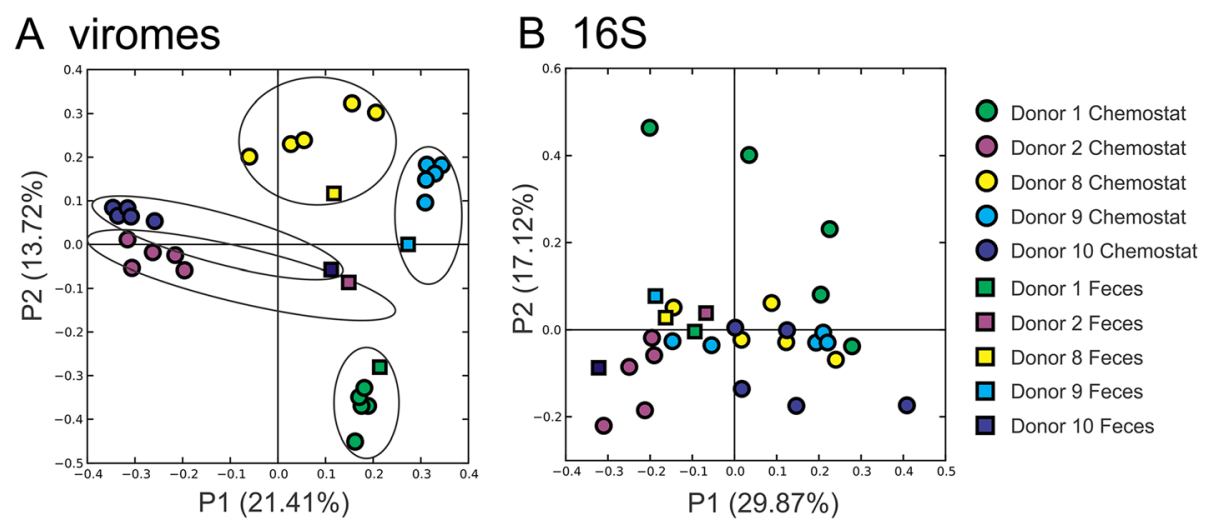

Fig. 6 Principal coordinates analysis of beta diversity present in the viromes based on patterns of similar contigs between each virome (a) and bacteria by 165 rRNA genes (b) of each subject and sample type. Fecal samples are represented by squares, and chemostat viromes are represented by circles. In $\mathbf{a}$, ovals are drawn around the data points for each individual donor

where the lowest evenness value of 0.1 resulted in the highest overestimates of diversity (Additional file 3: Figure S16). For evenness values of $0.33-0.9$, the percentage differences between the HVDI and the Shannon Index were $12 \%$ or lower and were highly consistent across the spectrum. These data indicate that the HVDI provided estimates of viral diversity similar to those of the Shannon Index and demonstrated that overestimates of viral diversity by the HVDI across different evenness levels were consistent and predictable.

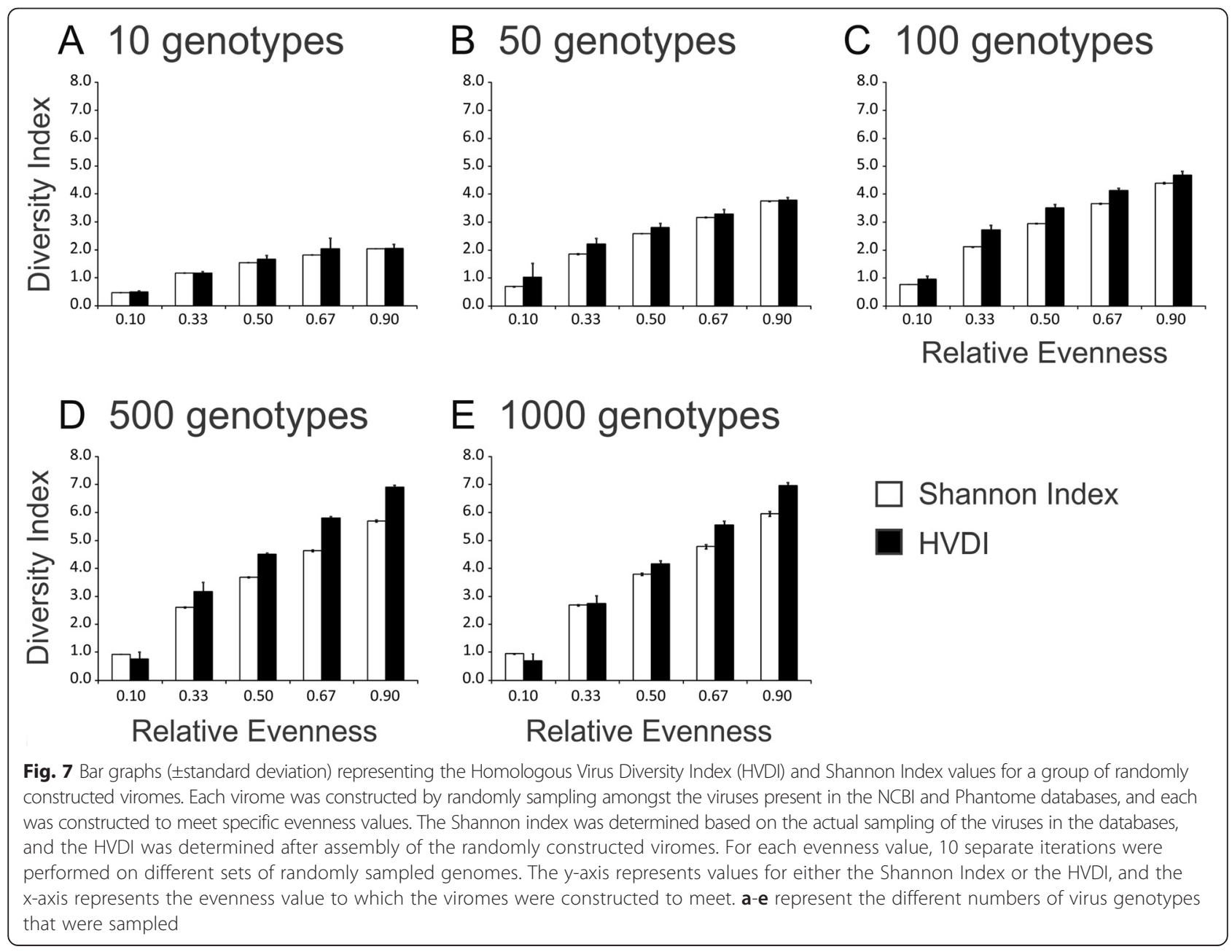


We next used the HVDI to perform rarefaction analysis to determine whether the viruses in the viromes had been adequately sampled and as a measure of whether the richness of viruses differed substantially between fecal and cultured communities. In this case, we calculated the HVDI based on the Chao1 index [48] because it penalizes more heavily for the presence of the rarer viral contigs in each sample. We found that there was no association between the sample type and the richness within the viral communities and that the diversity estimates approached asymptote in many cases, indicating that little additional viral diversity would have been identified through further sampling (Additional file 3: Figure S17).

We next compared the results of the HVDI using the Shannon Index to investigate whether the diversity of the viral component of cultured communities was similar to that from the feces in each subject. We found that for all subjects, viral diversity in the cultured communities changed as a function of time. For example, in donor \#2, viral diversity generally increased from day 4 to day 24 , while viral community diversity generally decreased in donor \#9. For donors \#1, \#2, and \#10, the diversity present in cultured communities on day 24 was highly similar to that in the feces of each subject (Fig. 8a). For donors \#8 and \#9, the diversity in cultured communities diminished significantly by day 18 which continued through day 24 . Neither of the day 24 viromes in donors \#8 or \#9 approximated the diversity present in the fecal viral communities. Evenness values for all subjects and time points were highly consistent with the HVDI data (Fig. 8b). Most of the evenness estimates ranged from 0.2 to 0.6 , which represents the range which the HVDI likely is to overestimate diversity by approximately $12 \%$ (Additional file 3: Figure S16). The evenness values for viral communities in donors \#8 and \#9 diminished significantly, which suggests that these communities were populated by fewer viruses that represented the majorities of the population. We repeated the virome preparations from the cultured communities on days 24 from donors \#8 and \#9 and similar results to those observed were found, indicating that there likely was a significant drop in viral diversity in these chemostat cultures (data not shown). Interestingly, the number of FSPs was not different for these cultured communities in donors $\# 8$ and $\# 9$, so the differences in evenness cannot be attributed to fewer total viruses being present. Alpha diversity for the bacteria as determined by the Shannon Index increased from day 4 to 24 and more closely approximated the feces by day 24 (Additional file 3: Figure S18). These data suggest that the relative loss of viral diversity in the chemostat cultures was not due an overall loss of bacterial diversity.

\section{Discussion}

Human body surfaces are home to large populations of viruses, whose role as members of human microbial communities is not well understood. In some environments, viral communities have been shown to be involved in driving bacterial diversity, yet no such data exists for human viral communities. We know that many of the phages that populate these communities are highly persistent and carry numerous pathogenic gene functions such as antibiotic resistance, which could help to shape bacterial community membership and its response to certain perturbations. Measuring their effects in humans is not without its challenges, which include the need to use drugs such as antibiotics, the need to reduce confounders such as diet variability, and the need to improve compliance with study protocols. The development of ecosystems that can approximate the dynamic interactions between phage communities and their cellular hosts can greatly reduce the reliance on human subjects in the characterization of human microbial communities.

Each virome in this study was subjected to MDA amplification due to the small amounts of viral DNA recovered from each donor. MDA amplification is known to introduce biases into sequence data [49], and it is unclear how MDA amplification biases could have affected these viromes. Many of the biases introduced often result when relatively low levels of starting DNA are utilized for amplification, which could have occurred in some of the viromes in this study. The relative conservation of viral genotypes across different time points in the chemostat cultures (Fig. 3) suggests that if MDA amplification biases affected the analysis of the viromes, the effects may have been relatively uniform across time points. The relative abundances of Microviridae, however, in the feces of four of the five subjects, may have been overrepresented through the use of MDA, as has previously been described [49]. The absence of microviruses in the chemostat cultures suggests that their bacterial host species may not be well represented in chemostat cultures.

Different bacteriophage families often have different lifestyles. Because phages can be significant drivers of diversity in different ecosystems [3-5, 11, 12, 39, 50], we examined whether there were phages with significant sequence similarities to known phage families in our cultured viral communities. Caudoviruses are the phage families most often found in our prior analyses of human oral viral communities $[9,10]$ and were commonly identified in the viral cultures in this study (Additional file 3: Figure S5). Of the different types of caudoviruses, siphoviruses generally have lysogenic lifestyles, while podoviruses and myoviruses more often have lytic lifestyles. We identified similar sequences to each of these viral families in our analysis of cultured viral communities, which strongly suggests that both primarily lytic and lysogenic phages were present. Not all myoviruses and podoviruses, however, have lytic lifestyles, as demonstrated by the presence of Enterobacteria phages FIAA91ss, IME10 (Fig. 2), and HK620 (Additional file 3: Figure S4), 

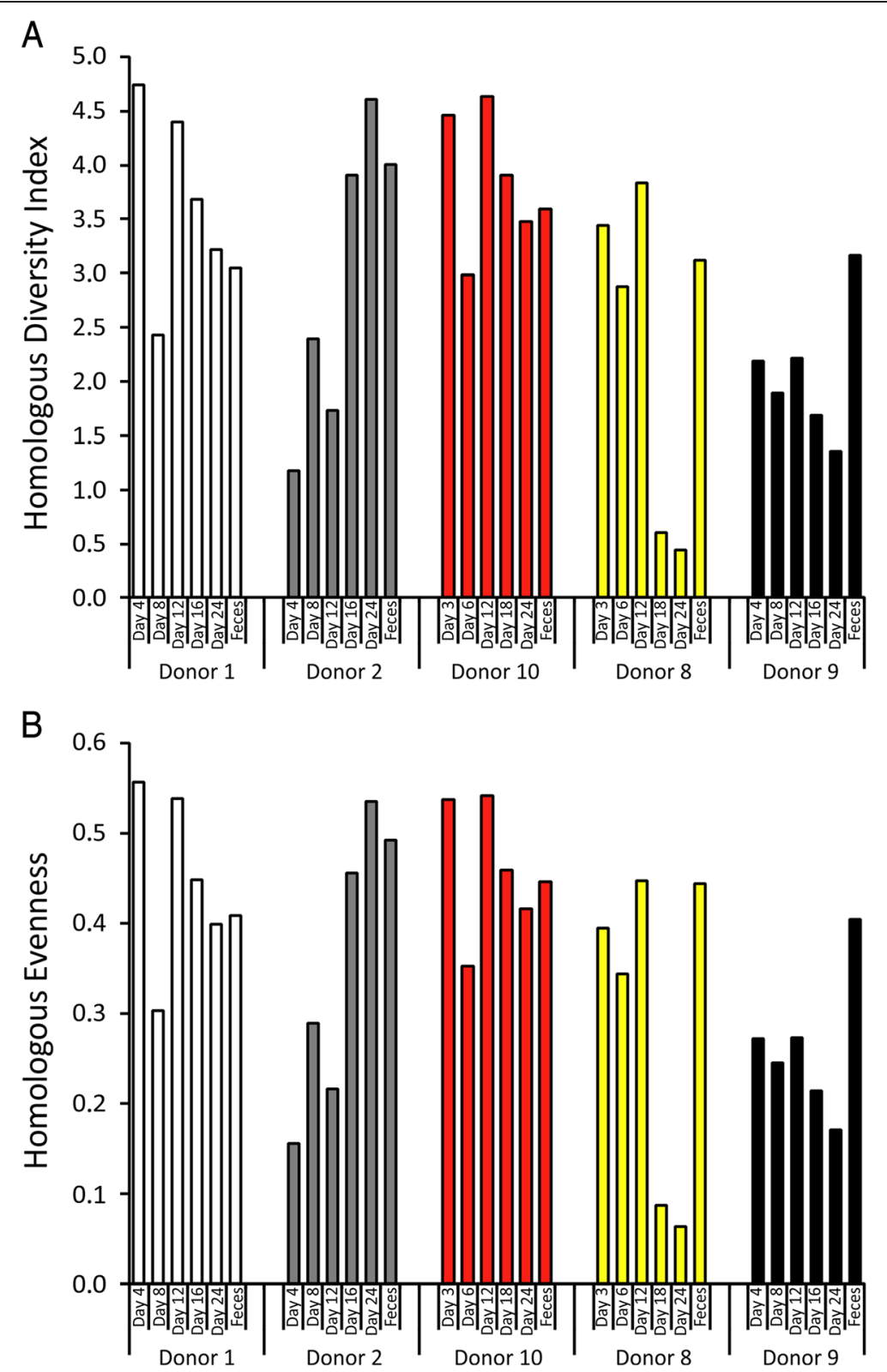

Fig. 8 Bar graphs representing the Homologous Virus Diversity Index (a) and Homologous Evenness Index (b) for all subjects. The $y$-axis represents diversity, and on the x-axis each bar from left to right represents day of culture. The last bar for each donor represents the fecal viromes

which have predicted gene functions that indicate their probable lysogenic lifestyles. The presence of each of these caudovirus families indicates that each of these virus types is viable in cultured communities. We found reads matching Enterobacteria phages FIAA91ss, IME10, and HK620 in the husband and wife but not in their daughter or the other donors. This suggests that these viruses were transmitted between husband and wife, but not to their offspring, potentially due to a lack of suitable host bacteria in the daughter.

Because of the high diversity in human phage communities and the varying relationships between host and phages, the relative abundances of phages do not necessarily reflect those of their host bacteria. This phenomenon was demonstrated in a prior study of human oral viral communities [39] and in a more recent study in the guts of humans with inflammatory bowel disease [51]. The data in this study support this finding, as the relative abundances of phage BLASTX hits from chemostat cultures and feces (Fig. 5) were not reflective of the relative abundances of the bacteria (Additional file 3: Figure S14). While BLASTX hit profiles do not necessarily represent taxonomic classifications, the abundances of phylum Bacteroidetes found in the phage BLASTX profiles and the 16S rRNA gene profiles 
suggest that many phages parasitizing these bacteria were present in the feces and chemostat cultures. Few Proteobacteria were identified through analysis of $16 \mathrm{~S}$ rRNA genes (Additional file 3: Figure S14), yet the abundance of phage hits to Proteobacteria was high (Fig. 5). These results may have been influenced by an unequal representation of phage from Proteobacteria in available databases, where a high relative abundance of phage from Proteobacteria renders us more likely to identify hits to Proteobacteria even when Proteobacteria are not the hosts of these phage.

Our prior studies of human viral communities have demonstrated that diversity in these communities is generally overestimated [10]. In that study, we found numerous different contigs that could not assemble, which actually belonged to the same phages. We developed a method to reduce the overestimation of viral diversity by finding high levels of similarity amongst these contigs and assigning their contig spectra to the same virus genotype. We do not believe that in every case high levels of similarity necessarily represent the same virus; however, utilizing this technique allowed us to estimate viral community diversity within $12 \%$ of the actual diversity in our simulated communities across most evenness levels. This technique allowed for us to provide estimates of sequencing depth adequacy as well as comparisons of diversity amongst fecal and cultured viral communities. While the data indicate that most of the viral diversity in these communities could be identified by sequencing <20,000 reads (Additional file 3: Figure S15), the greater number of reads used in this study was necessary to assemble more reliable contig spectra. The rarefaction analyses strongly suggested that further sequencing would not have added substantially to the diversity estimates present in cultured or fecal viral communities. We believe that tools such as the HVDI add to available methodologies for examining viral community diversity and will be of great utility in understanding the responses of viral communities to perturbations.

One of the major goals of this work was to examine how closely cultured communities might approximate human indigenous phage communities. Identifying numerous FSPs and many reconstructed phages in the cultured communities (Figs. 2 and 4, and Additional file 3: Figures S10, S11, S12 and S13) strongly suggests that chemostat culture systems are fully capable of supporting robust phage communities. Chemostat cultured communities have been known to support the viability of individual phages [30]; however, the data presented here indicate that chemostat cultures can support entire communities of phages as well. Our focus on contigs rather than virome reads allowed us to characterize $94.9 \%$ of the viromes (far greater than often is reported in virome studies), which we believe provides a broad overview of the phage present in chemostat cultures and feces. The significant drop in diversity and evenness in the viromes in donors $\# 8$ and $\# 9$ by day 18 cannot be attributed to a drop in bacterial diversity (Additional file 3: Figure S14), and the BLASTX profiles in these subjects (Fig. 5) suggest that while diversity was diminished, it likely was diminished across different bacterial host lineages. While these cultured communities do not perfectly approximate the diversity of phages in the human gut, as evidenced by the generally higher similarities within chemostat viromes compared with fecal viromes (Fig. 3), the ability to approximate diversity in some human viromes (Fig. 8) should prove useful for furthering our understanding of host/phage interactions in humans. We did not perform detailed comparisons of phages and their putative bacterial hosts in each donor because BLASTX is generally considered insufficient to accurately predict the hosts of each phage.

\section{Conclusions}

By establishing phage communities that have some similarities to those found on human body surfaces, several important questions can be addressed. These questions include the following: (1) what is the role of phages in driving the diversity of the bacteria in human microbial communities?, (2) how do perturbations such as antibiotics impact human phage communities?, (3) how does the sharing of phage communities between individuals impact microbial community membership?, and (4) what are the dynamics of most phages as members of human microbial communities? While there are quantifiable differences between the phage communities present in feces and cultured communities, there also are many similarities. The relative number of FSPs in both sample types are similar, the profiles of beta diversity strongly suggest a conservation of some phage community members across fecal and cultured communities, and the relative levels of phage diversity between communities in some subjects were highly similar. By establishing cultured phage communities, we can begin to understand the role and contributions of phages to human microbial communities.

\section{Methods}

\section{Ethics, consent, and permissions}

Human subject recruitment and enrollment in this study was approved by The Research Ethics Board of the University of Guelph REB\#13AP008 and 10JL002. Each subject signed a written informed consent confirming their willingness to participate in this study.

\section{Human subjects}

Five healthy donors provided fecal samples: donor \#1 (male, 44 years old), donor \#2 (female, 41 years old), donor \#8 (female, 26 years old), donor \#9 (male, 25 years old), and donor \#10 (female, 7 years old). Donors \#1, \#2, and \#10 were a co-habiting family unit of father, mother, 
and child, respectively. Donors \#8 and \#9 were unrelated. None of the donors had a recent history of antibiotic treatment for 9 months prior to sampling. Each donor provided at least $5 \mathrm{~g}$ of fresh fecal samples for the chemostat cultures. Donor \#10 provided a sample in the home environment which was immediately wrapped in plastic cling wrap to exclude air and then frozen at $-20{ }^{\circ} \mathrm{C}$ for overnight transportation to the lab. The remaining donors provided fresh samples.

\section{Chemostat cultures}

All samples were placed immediately into an anaerobic chamber $\left(90 \% \mathrm{~N}_{2}, 5 \% \mathrm{CO}_{2}, 5 \% \mathrm{H}_{2}\right)$ upon receipt at the lab; for the fresh samples, this was within $30 \mathrm{~min}$ of collection. The sample from donor \#10 was allowed to thaw in the chamber. A modified Infors Multifors system was used to run chemostat cultures modeling the human distal colon environment, as described by McDonald et al. [29]. A $10 \%(w / v)$ slurry was prepared from each donor by homogenizing feces in pre-reduced growth medium using a stomacher. For every $1 \mathrm{~L}$ of medium, the following components were included in the growth medium: peptone water, 2 g; yeast extract, $2 \mathrm{~g}, \mathrm{NaHCO} 3,2 \mathrm{~g} ; \mathrm{CaCl} 2,0.01 \mathrm{~g}$; pectin (from citrus), $2 \mathrm{~g}$; xylan (from beechwood), $2 \mathrm{~g}$; arabinogalactan, $2 \mathrm{~g}$; starch (from wheat, unmodified), $5 \mathrm{~g}$; casein, $3 \mathrm{~g}$; inulin (from Dahlia tubers), $1 \mathrm{~g}$; bile salts, $0.5 \mathrm{~g}$ $\mathrm{NaCl}, 0.1 \mathrm{~g}$; L-cysteine $\mathrm{HCl}, 0.5 \mathrm{~g}$; K2HPO4, $0.04 \mathrm{~g}$; $\mathrm{KH} 2 \mathrm{PO} 4,0.04 \mathrm{~g}$; MgSO4, $0.01 \mathrm{~g}$; hemin, $0.005 \mathrm{~g}$; and menadione, $0.001 \mathrm{~g}$ (all components from Sigma Aldrich). Growth media was stored at $4{ }^{\circ} \mathrm{C}$ until use for a maximum of 2 weeks. The fecal slurry in growth medium was then centrifuged to remove large particles [57], and the supernatant was used as the inoculum for each experiment by adding $100 \mathrm{~mL}$ into $300 \mathrm{~mL}$ of sterile growth medium in each vessel. The $\mathrm{pH}$ within each vessel was then adjusted to 6.9-7.0, and the cultures gently and continually agitated and maintained at $37{ }^{\circ} \mathrm{C}$. Vessels were run in batch mode for $24 \mathrm{~h}$ to allow inoculum recovery time (adjustment from in vivo to in vitro conditions) and to avoid wash-out. The pumps were then switched on, and the retention rate set to $16.67 \mathrm{~mL} / \mathrm{h}^{-1}$ with constant sparging of $\mathrm{O}_{2}$ free $\mathrm{N}_{2}$ gas to maintain positive pressure and anaerobiosis. Each chemostat vessel was sampled daily by aseptically removing $4 \mathrm{~mL}$ of culture directly from the vessel contents, and all samples were archived at $-80{ }^{\circ} \mathrm{C}$. Aliquots of the original fecal samples also were archived at $-80{ }^{\circ} \mathrm{C}$ for virome processing.

\section{Preparation and sequencing of viromes}

Fecal viromes were prepared by diluting $0.4 \mathrm{~g}$ of feces in $4 \mathrm{~mL}$ of SM buffer. The fecal samples were vortexed vigorously for $40 \mathrm{~min}$ to separate viral particles, spun at $4000 \times g$ for $10 \mathrm{~min}$ to pellet the remaining solid material, and the supernatant was treated in an identical manner to that of the chemostat cultures. A small portion $(10 \mu \mathrm{L})$ of the supernatant from each donor was resuspended in $190 \mu \mathrm{L}$ of $0.02-\mu \mathrm{m}$ filtered PBS and their counts per milliliter determined by epifluorescence microscopy [38]. Chemostat samples and fecal supernatants were filtered sequentially using 0.45 and $0.2 \mu \mathrm{m}$ filters (VWR, Radnor, PA) to remove cellular and other debris and then purified on a cesium chloride gradient according to previously described protocols [39]. Only the fraction with a density corresponding to most known bacteriophages [52] was retained, further purified on Amicon YM-100 protein purification columns (Millipore, Inc., Bellerica, MA), treated with DNase I, and subjected to lysis and DNA purification using the Qiagen UltraSens Virus kit (Qiagen, Valencia, $\mathrm{CA})$. Recovered DNA was screened for the presence of contaminating bacterial nucleic acids by quantitative $16 \mathrm{~S}$ rRNA gene PCR using primers 8F (AGAGTTTGATC CTGGCTCAG) and 357R (CTGCTGCCTYCCGTA) in Power SYBR Green PCR Mastermix (Thermo Fisher Scientific, Carlsbad, CA). No products were detected in any of the viromes after 35 cycles, which does not exclude the presence of contaminating bacterial nucleic acids but indicates that they were not present at dominant levels. Resulting DNA was amplified using GenomiPhi Hy MDA amplification (GE Healthcare, Pittsburgh, PA), fragmented to roughly 200 to $400 \mathrm{bp}$ using a Bioruptor (Diagenode, Denville, NJ), and utilized as input to create libraries using the Ion Plus Fragment Library Kit according to manufacturer's instructions. Libraries then were sequenced using 314 or 316 chips on an Ion Torrent Personal Genome Machine (PGM; Life Technologies, Grand Island, NY) [40] producing an average read length of approximately 215 bp for each sample.

\section{Analysis of viromes}

Due to the error rate of semiconductor sequencing [40], we trimmed each read according to modified Phred scores of 0.5 using CLC Genomics Workbench 8.01 (CLC bio USA, Cambridge, MA), removed any low complexity reads with $\geq 8$ consecutive homopolymers, and removed any reads with substantial length variation $(<50$ nucleotides or $>300$ nucleotides) or ambiguous characters prior to further analysis. Each virome was screened for contaminating bacterial and human nucleic acids using BLASTN analysis (E-value $<10^{-5}$ ) against the Ribosomal Database Project 16S rRNA genes database [53] and the human reference database available at $\mathrm{ftp} / / \mathrm{ftp}$.ncbi.nlm.nih.gov/ genomes/H_sapiens/. Any reads with significant sequence similarities to human sequences were removed prior to further analysis. Length and GC content variation amongst contigs was assessed using Box and Whiskers plots created using Microsoft Excel 2007 (Microsoft Corp., Redman, WA). All reads were assembled using CLC Genomics Workbench 8.01 based on $98 \%$ identity with a minimum 
of $50 \%$ read overlap, which were more stringent than criteria developed to discriminate between highly related viruses [54]. The assembly method works by using a de Bruijn graph technique and various word lengths, similar to that used in the assembler Velvet [55]. We also used MetaVelvet [41] and IDBA-UD [42] in the construction of contigs, but the CLC assembler produced fewer contigs of higher mean lengths with greater N50 values (Additional file 2: Table S2). Because the shortest reads were 50 nucleotides, the minimum tolerable overlap was 25 nucleotides, and the average overlap was no less than 100 nucleotides depending on the characteristics of each virome. The consensus sequence for each contig was constructed according to majority rule, and any contigs $<200$ nucleotides or with ambiguous characters were removed prior to further analysis. Read mapping of viromes to a combined database of viruses (www.phantome.org; ftp://ftp.ncbi.nih. gov/genomes/Viruses/) was performed using CLC Genomics Workbench 8.01 (CLC bio USA, Cambridge, MA) and were mapped using $98 \%$ identity over a minimum of $50 \%$ of the read length. For each donor, we also utilized a separate technique for assembly by constructing global assemblies from all contigs from all time points using $98 \%$ identity over a minimum of $50 \%$ overlap. Viral contigs were analyzed using FGenesV (Softberry Inc, Mount Kisco, NY) for ORF prediction and individual ORFs analyzed using BLASTP analysis against the NCBI non-redundant database $\left(E-v a l u e ~<10^{-5}\right)$. If the best hit was to a gene with no known function, lower level hits were used for the annotation as long as they had known putative function and still met the E-value cutoff $\left(10^{-5}\right)$.

Contigs were annotated using BLASTX against the NCBI NR database with an E-value cutoff value of $10^{-5}$. Specific viral sequences were identified by parsing BLASTX results for known viral genes including replication, structural, transposition, restriction/modification, hypothetical, and other genes previously found in viruses for which the E-value was at least $10^{-5}$. Each individual virome contig was annotated using this technique; however, if the best hit for any portion of the contig was to a gene with no known function, lower level hits were used as long as they had known function and still met the E-value cutoff. The annotation data were compiled by the number of reads used to assemble each contig for each subject and used to determine the relative proportions of contigs that contained viral sequences. The phyla of the BLASTX best hits for each annotated contig were used to create profiles in each donor and sample type. We utilized the average coverage in the assemblies of each contig to determine the relative abundance profiles of different phyla to compensate for viruses that may be more abundant than others. This technique prevented reads involved in the assembly of the same virus contigs from being assigned to different putative host phyla based on different BLASTX similarities. Determination of the relative abundances of virus families was determined by BLASTX analysis of the SEED database using MG-RAST [43].

Analysis of shared sequence similarities present in each virome was performed by creating custom BLAST databases for each virome, comparing each database with all other viromes using BLASTN analysis (E-value $<10^{-10}$ ). Principal coordinates analysis (PCOA) was performed on virome contigs with Bray Curtis distances using Qiime [56]. We also utilized a separate technique for assembly by constructing global assemblies from all contigs from all subjects and time points using $98 \%$ identity over a minimum of $50 \%$ overlap. The contribution of each subject and time point to each assembly was assessed and utilized to determine relative persistence of phages over time in the chemostat cultures and as input for PCOA and for heat matrix analysis using Microsoft Excel. We also used the profiles of BLASTX best hits amongst all subjects and time points as input for PCOA. Beta diversity based on Bray Curtis distances was used as input for each PCOA.

\section{Analysis of 16S rRNA genes}

Genomic DNA was prepared from the feces of each subject and time point using the Qiagen QIAamp DNA Stool Mini kit (Qiagen, Valencia, CA). We amplified the bacterial 16S rRNA gene V1-V2 hypervariable region using the forward primer 8F fused with the Ion Torrent Adaptor A sequence and one of 23 unique 10 base pair barcodes and reverse primer 357R fused with the Ion Torrent Adaptor P1 from the each donor and sample type [57]. PCR reactions were performed using Platinum PCR High Fidelity SuperMix (Invitrogen, Carlsbad, CA) with the following cycling parameters: $94^{\circ} \mathrm{C}$ for $10 \mathrm{~min}$, followed by 30 cycles of $94{ }^{\circ} \mathrm{C}$ for $30 \mathrm{~s}, 53{ }^{\circ} \mathrm{C}$ for $30 \mathrm{~s}, 72{ }^{\circ} \mathrm{C}$ for $30 \mathrm{~s}$, and a final elongation step of $72{ }^{\circ} \mathrm{C}$ for $10 \mathrm{~min}$. Resulting amplicons were purified on a $2 \%$ agarose gel stained with SYBR Safe (Invitrogen, Carlsbad, CA) using the MinElute PCR Purification kit (Qiagen, Valencia, CA). Amplicons were further purified with Ampure XP beads (Beckman-Coulter, Brea, $\mathrm{CA}$ ), and molar equivalents were determined for each sample using a Bioanalyzer 2100 HS DNA Kit (Agilent Technologies, Santa Clara, CA). Samples were pooled into equimolar proportions and sequenced on 314 chips using an Ion Torrent PGM according to manufacturer's instructions (Life Technologies, Grand Island, NY) [40]. Resulting sequence reads were removed from the analysis if they were $<180$ nucleotides, had any barcode or primer errors, contained any ambiguous characters, or contained any stretch of $>8$ homopolymers. Sequences were assigned to their respective samples based on a 10-nucleotide barcode sequence and were analyzed further using the Qiime pipeline [56]. Briefly, representative OTUs from each set were 
chosen at a minimum sequence identity of $97 \%$ using UClust [58] and aligned using PyNast [59] against the Greengenes database [60]. Multiple alignments then were used to create phylogenies using FastTree [61], and taxonomy was assigned to each OTU using the RDP classifier $[62,63]$. PCOA was performed based on beta diversity using weighted Unifrac distances [64].

\section{Statistical analysis}

To assess whether virome contigs had significant overlap within or between donors and sample types, we performed a permutation test based on resampling (10,000 iteration). We simulated the distribution of the fraction of virome sequences with significant similarities from two different sample types within a donor. For each set, we computed the summed fraction of sequences with significant similarities using 1000 random contigs between different donors, and from these computed an empirical null distribution of our statistic of interest (the fraction of shared similar sequences). The simulated statistics within each donor were referred to the null distribution of inter-donor comparisons, and the $p$ value was computed as the fraction of times the simulated statistic for each exceeded the observed statistic. For analysis of fecal versus cultured viromes, a randomly chosen donor from the cultured virome group was compared with a randomly selected donor from the fecal group to determine the null distribution of the fraction of shared contigs. We then estimated the fraction of shared sequences with significant similarities from randomly chosen donors within the cultured virome group and compared with the empirical null distribution from simulated inter-group values. Intra-subject comparisons were excluded from this analysis. We estimated the $p$ value based on the fraction of times the intra-group statistic exceeded that for the null statistic.

\section{Homologous virus diversity index and virome construction}

To measure alpha diversity in the viral communities, we utilized a technique termed the Homologous Virus Diversity Index [47]. The technique is based on finding high levels of similarity amongst contigs within viromes that potentially belong to the same or a highly similar viral genotype but were placed into separate contigs due to the limitations of the assembly process [10]. We validated the technique by constructing viromes composed of randomly selected viruses amongst both the NCBI (ftp://ftp.ncbi.nlm. nih.gov/genomes/Viruses/) and Phantome viral databases (www.phantome.org). First, a random set of viruses was chosen from amongst the databases for each virome, and viromes were constructed that contained 10 viruses, 50 viruses, 100 viruses, 500 viruses, and 1000 viruses. We constructed 10 different viromes at each level of different viral genotypes. The reads in each virome were of mean length
$200 \mathrm{bp}$ and were chosen randomly across the genomes of each virus. The shortest reads were $150 \mathrm{bp}$, and the longest reads were $250 \mathrm{bp}$ in each constructed virome. Each virome also was constructed to meet specific evenness requirements, where an evenness of close to 1 would indicate a population consisting of different viruses all of similar relative abundances, and an evenness close to 0 would indicate a population where the relative abundance of a few viruses are much greater than others. We utilized evenness as criteria to construct the viromes because higher evenness values would prove more difficult for the assembly process. For each constructed virome, we created contig spectra by counting the number of reads assigned to each different virus in the virome. The contig spectra then were used as surrogates for population structures in determination of the Shannon Index [46]. The actual evenness values for each constructed virome were determined using the equation $H / \ln (S)$, where $H$ is the Shannon Index value and $\mathrm{S}$ is the total number of viruses in each virome. We also determined the HVDI for each constructed virome by assembling the reads using $98 \%$ identity over a minimum of $50 \%$ of the read length using CLC Genomics Workbench 8.01 (CLC bio USA, Cambridge, MA). The resulting contigs then were subjected to BLASTN analysis against a database of contigs from the exact same subject, and contigs with high degrees of similarity (E-value $<10^{-20}$ ) over $50 \%$ of the length of the shorter contig were assigned to the same viral genotype. The spectra from each individual contig that were assigned to the same genotype were added to a corrected contig spectrum for each subject and those spectra used as inputs for the Shannon Index [46] to calculate the HVDI. The actual Shannon Index values for each constructed virome were compared to the results of the HVDI in each case, and the HVDI estimates exceeded the Shannon Index by approximately $12 \%$ across most evenness levels. Rarefactions were determined for each donor and time point by randomly sampling up to 20,000 reads. The randomly sampled reads then were assigned to their respective contigs by comparing them with the corrected contig spectra generated after the BLASTN analysis. We then constructed new contig spectra based on the sampled reads and their assignments to different viral genotypes and used those spectra in calculations of the HVDI. For the rarefactions, we calculated the HVDI using the Chao1 index [48], which utilizes the relative numbers of viral contigs created from only a single read (singleton) or from only two reads (doubleton) as input.

\section{Availability of data and materials}

All sequences including viromes and 16S rRNA genes are available for download in the MG-RAST database (metagenomics.anl.gov/) under the project "Chemostat," or project \#10563. 


\section{Additional files}

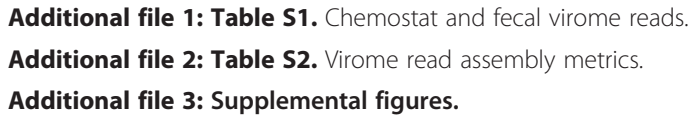

\section{Competing interests}

The authors declare that they have no competing interests.

\section{Authors' contributions}

DTP and EAV conceived and designed the experiments. TSR, ML, MCD, IHLB, JAKM, and NB performed the experiments. DTP, TS-R, and EAV analyzed the data. MCD, IHLB, JAKM, and EAV contributed reagents. DTP, EA-V, and TSR wrote the manuscript.

\section{Acknowledgements}

Supported by the Burroughs Wellcome Fund grant to DTP, an Ontario Ministry of Agriculture Food and Rural Affairs (OMAFRA)/University of Guelph partnership grant, a Physician's Services Incorporated grant, and a US Department of Defense CDMRP grant (W81XWH-13-1-0376) to E.A-V.

\section{Author details}

${ }^{1}$ Department of Pathology, University of California, 9500 Gilman Drive, MC 0612, La Jolla, CA 92093-0612, USA. ²Department of Molecular and Cellular Biology, University of Guelph, Guelph, Ontario, Canada. ${ }^{3}$ Department of Biology, San Diego State University, San Diego, CA, USA. ${ }^{4}$ Department of Medicine, University of California, San Diego, CA, USA.

\section{Received: 28 August 2015 Accepted: 27 October 2015}

\section{Published online: 09 November 2015}

\section{References}

1. Hofer U. Viral evolution: variation in the gut virome. Nat Rev Microbiol. 2013;11:596

2. Haynes M, Rohwer F. The human virome. In: Metagenomics of the human body. New York: Springer; 2011. p. 63-77.

3. Minot S, Bryson A, Chehoud C, Wu GD, Lewis JD, Bushman FD. Rapid evolution of the human gut virome. Proc Natl Acad Sci U S A. 2013;110:12450-5.

4. Reyes A, Haynes M, Hanson N, Angly FE, Heath AC, Rohwer F, et al. Viruses in the faecal microbiota of monozygotic twins and their mothers. Nature. 2010;466:334-8

5. Minot S, Sinha R, Chen J, Li H, Keilbaugh SA, Wu GD, et al. The human gut virome: inter-individual variation and dynamic response to diet. Genome Res. 2011;21:1616-25.

6. Naidu M, Robles-Sikisaka R, Abeles SR, Boehm TK, Pride DT. Characterization of bacteriophage communities and CRISPR profiles from dental plaque. BMC Microbiol. 2014;14:175.

7. Labrie SJ, Samson JE, Moineau S. Bacteriophage resistance mechanisms. Nat Rev Microbiol. 2010;8:317-27.

8. Pride DT, Sun CL, Salzman J, Rao N, Loomer P, Armitage GC, et al. Analysis of streptococcal CRISPRs from human saliva reveals substantial sequence diversity within and between subjects over time. Genome Res. 2011;21:126-36.

9. Ly M, Abeles SR, Boehm TK, Robles-Sikisaka R, Naidu M, Santiago-Rodriguez $\mathrm{T}$, et al. Altered oral viral ecology in association with periodontal disease. MBio. 2014;5:e01133-14.

10. Abeles SR, Robles-Sikisaka R, Ly M, Lum AG, Salzman J, Boehm TK, et al. Human oral viruses are personal, persistent and gender-consistent. ISME J. 2014:8:1753-67.

11. Foulongne V, Sauvage V, Hebert C, Dereure O, Cheval J, Gouilh MA, et al. Human skin microbiota: high diversity of DNA viruses identified on the human skin by high throughput sequencing. PLoS One. 2012;7:e38499.

12. Willner D, Furlan M, Haynes M, Schmieder R, Angly FE, Silva J, et al. Metagenomic analysis of respiratory tract DNA viral communities in cystic fibrosis and non-cystic fibrosis individuals. PLoS One. 2009;4:e7370.

13. Heldal M, Bratbak G. Production and decay of viruses in aquatic environments. Mar Ecol Prog Ser. 1991;72:205-12.

14. Doolittle M, Cooney J, Caldwell D. Lytic infection of Escherichia coli biofilms by bacteriophage T4. Can J Microbiol. 1995;41:12-8.
15. Fortier LC, Sekulovic O. Importance of prophages to evolution and virulence of bacterial pathogens. Virulence. 2013;4:354-65.

16. Ventura M, Sozzi T, Turroni F, Matteuzzi D, van Sinderen D. The impact of bacteriophages on probiotic bacteria and gut microbiota diversity. Genes Nutr. 2011;6:205-7

17. Suttle CA. Marine viruses-major players in the global ecosystem. Nat Rev Microbiol. 2007:5:801-12.

18. Dinsdale EA, Edwards RA, Hall D, Angly F, Breitbart M, Brulc JM, et al. Functional metagenomic profiling of nine biomes. Nature. 2008;452:629-32.

19. Clokie MR, Millard AD, Letarov AV, Heaphy S. Phages in nature. Bacteriophage. 2011;1:31-45.

20. Macfarlane GT, Macfarlane S. Models for intestinal fermentation: association between food components, delivery systems, bioavailability and functional interactions in the gut. Curr Opin Biotechnol. 2007;18:156-62.

21. Zoetendal EG, Akkermans AD, De Vos WM. Temperature gradient gel electrophoresis analysis of 165 rRNA from human fecal samples reveals stable and host-specific communities of active bacteria. Appl Environ Microbiol. 1998;64:3854-9.

22. Russell SL, Gold MJ, Reynolds LA, Willing BP, Dimitriu P, Thorson L, et al. Perinatal antibiotic-induced shifts in gut microbiota have differential effects on inflammatory lung diseases. J Allergy Clin Immunol. 2014;135(1):100-9. doi:10.1016/j.jaci.2014.06.027.

23. Ellekilde M, Selfjord E, Larsen CS, Jakesevic M, Rune I, Tranberg B, et al. Transfer of gut microbiota from lean and obese mice to antibiotic-treated mice. Sci Rep. 2014:4:5922.

24. Lu N, Hu Y, Zhu L, Yang $X$, Yin Y, Lei F, et al. DNA microarray analysis reveals that antibiotic resistance-gene diversity in human gut microbiota is age related. Sci Rep. 2014;4:4302.

25. Perez-Cobas AE, Artacho A, Knecht H, Ferrus ML, Friedrichs A, Ott SJ, et al. Differential effects of antibiotic therapy on the structure and function of human gut microbiota. PLoS One. 2013;8:e80201.

26. Hu Y, Yang X, Qin J, Lu N, Cheng G, Wu N, et al. Metagenome-wide analysis of antibiotic resistance genes in a large cohort of human gut microbiota. Nat Commun. 2013:4:2151.

27. Brace C, Gloor GB, Ropeleski M, Allen-Vercoe E, Petrof EO. Microbial composition analysis of Clostridium difficile infections in an ulcerative colitis patient treated with multiple fecal microbiota transplantations. J Crohns Colitis. 2014;8:1133-7.

28. Freter $\mathrm{R}$, Brickner $\mathrm{H}$, Botney M, Cleven D, Aranki A. Mechanisms that control bacterial populations in continuous-flow culture models of mouse large intestinal flora. Infect Immun. 1983;39:676-85.

29. Reyes A, Wu M, McNulty NP, Rohwer FL, Gordon Jl. Gnotobiotic mouse model of phage-bacterial host dynamics in the human gut. Proc Natl Acad Sci U S A. 2013;110:20236-41.

30. McDonald JA, Schroeter K, Fuentes S, Heikamp-Dejong I, Khursigara CM, de Vos WM, et al. Evaluation of microbial community reproducibility, stability and composition in a human distal gut chemostat model. J Microbiol Methods. 2013:95:167-74.

31. Crowther GS, Chilton CH, Todhunter SL, Nicholson S, Freeman J, Baines SD, et al. Development and validation of a chemostat gut model to study both planktonic and biofilm modes of growth of Clostridium difficile and human microbiota. PLoS One. 2014;9:e88396.

32. Petrof EO, Gloor GB, Vanner SJ, Weese SJ, Carter D, Daigneault MC, et al. Stool substitute transplant therapy for the eradication of Clostridium difficile infection: "RePOOPulating" the gut. Microbiome. 2013;1:3.

33. Shahinas D, Silverman M, Sittler T, Chiu C, Kim P, Allen-Vercoe E, et al. Toward an understanding of changes in diversity associated with fecal microbiome transplantation based on 16S rRNA gene deep sequencing. MBio. 2012;3:5. doi:10.1128/mBio.00338-12.

34. Edlund A, Yang Y, Hall AP, Guo L, Lux R, He X, et al. An in vitro biofilm model system maintaining a highly reproducible species and metabolic diversity approaching that of the human oral microbiome. Microbiome. 2013;1:25.

35. Crowther GS, Chilton CH, Todhunter SL, Nicholson S, Freeman J, Baines SD, et al. Comparison of planktonic and biofilm-associated communities of Clostridium difficile and indigenous gut microbiota in a triple-stage chemostat gut model J Antimicrob Chemother. 2014;69:2137-47.

36. Fischer CR, Yoichi M, Unno H, Tanji Y. The coexistence of Escherichia coli serotype 0157:H7 and its specific bacteriophage in continuous culture. FEMS Microbiol Lett. 2004;241:171-7.

37. Mizoguchi K, Morita M, Fischer CR, Yoichi M, Tanji Y, Unno H. Coevolution of bacteriophage PP01 and Escherichia coli 0157:H7 in continuous culture. Appl Environ Microbiol. 2003;69:170-6. 
38. Noble RT, Fuhrman JA. Use of SYBR Green I for rapid epifluorescence counts of marine viruses and bacteria. Aquat Microb Ecol. 1998;14:113-8.

39. Pride DT, Salzman J, Haynes M, Rohwer F, Davis-Long C, White 3rd RA, et al. Evidence of a robust resident bacteriophage population revealed through analysis of the human salivary virome. ISME J. 2012;6:915-26.

40. Rothberg JM, Hinz W, Rearick TM, Schultz J, Mileski W, Davey M, et al. An integrated semiconductor device enabling non-optical genome sequencing. Nature. 2011:475:348-52.

41. Namiki T, Hachiya T, Tanaka H, Sakakibara Y. MetaVelvet: an extension of Velvet assembler to de novo metagenome assembly from short sequence reads. Nucleic Acids Res. 2012;40:e155.

42. Peng Y, Leung HC, Yiu SM, Chin FY. IDBA-UD: a de novo assembler for single-cell and metagenomic sequencing data with highly uneven depth. Bioinformatics. 2012;28:1420-8.

43. Meyer F, Paarmann D, D'Souza M, Olson R, Glass EM, Kubal M, et al. The metagenomics RAST server-a public resource for the automatic phylogenetic and functional analysis of metagenomes. BMC Bioinformatics. 2008:9:386.

44. Robles-Sikisaka R, Ly M, Boehm T, Naidu M, Salzman J, Pride DT. Association between living environment and human oral viral ecology. ISME J. 2013;7:1710-24.

45. Dutilh BE, Cassman N, McNair K, Sanchez SE, Silva GG, Boling L, et al. A highly abundant bacteriophage discovered in the unknown sequences of human faecal metagenomes. Nat Commun. 2014;5:4498.

46. Gotelli NJ, Colwell RK. Quantifying biodiversity: procedures and pitfalls in the measurement and comparison of species richness. Ecol Lett. 2001:4:379-91.

47. Santiago-Rodriguez TM, Ly M, Bonilla N, Pride DT. The human urine virome in association with urinary tract infections. Front Microbiol. 2015:6:14.

48. Chao A. Non-parametric estimation of the number of classes in a population. Scand J Stat. 1984;11:265-70.

49. Kim KH, Bae JW. Amplification methods bias metagenomic libraries of uncultured single-stranded and double-stranded DNA viruses. Appl Environ Microbiol. 2011;77:7663-8.

50. Willner D, Furlan M, Schmieder R, Grasis JA, Pride DT, Relman DA, et al. Metagenomic detection of phage-encoded platelet-binding factors in the human oral cavity. Proc Natl Acad Sci U S A. 2011;108 Suppl 1:4547-53.

51. Norman JM, Handley SA, Baldridge MT, Droit L, Liu CY, Keller BC, et al. Disease-specific alterations in the enteric virome in inflammatory bowel disease. Cell. 2015;160:447-60.

52. Murphy FA, Fauquet CM, Bishop DHL, Ghabrial SA, Jarvis AW, Martelli GP, et al. Virus taxonomy: sixth report of the international committee on taxonomy of viruses, vol. Supplement 10. New York: Springer; 1995.

53. Cole JR, Wang Q, Cardenas E, Fish J, Chai B, Farris RJ, et al. The ribosomal database project: improved alignments and new tools for rRNA analysis. Nucleic Acids Res. 2009:37:D141-5.

54. Breitbart M, Salamon P, Andresen B, Mahaffy JM, Segall AM, Mead D, et al. Genomic analysis of uncultured marine viral communities. Proc Natl Acad Sci U S A. 2002;99:14250-5.

55. Zerbino DR, Birney E. Velvet: algorithms for de novo short read assembly using de Bruijn graphs. Genome Res. 2008;18:821-9.

56. Caporaso JG, Kuczynski J, Stombaugh J, Bittinger K, Bushman FD, Costello EK, et al. QIIME allows analysis of high-throughput community sequencing data. Nat Methods. 2010;7:335-6.

57. Whiteley AS, Jenkins S, Waite I, Kresoje N, Payne H, Mullan B, et al. Microbial 165 rRNA lon Tag and community metagenome sequencing using the lon Torrent (PGM) Platform. J Microbiol Methods. 2012:91:80-8.

58. Edgar RC. Search and clustering orders of magnitude faster than BLAST. Bioinformatics. 2010;26:2460-1.

59. Caporaso JG, Bittinger K, Bushman FD, DeSantis TZ, Andersen GL, Knight R PYNAST: a flexible tool for aligning sequences to a template alignment. Bioinformatics. 2010:26:266-7.

60. DeSantis TZ, Hugenholtz P, Larsen N, Rojas M, Brodie EL, Keller K, et al. Greengenes, a chimera-checked 16S rRNA gene database and workbench compatible with ARB. Appl Environ Microbiol. 2006;72:5069-72.

61. Li W, Godzik A. Cd-hit: a fast program for clustering and comparing large sets of protein or nucleotide sequences. Bioinformatics. 2006;22:1658-9.
62. Price MN, Dehal PS, Arkin AP. FastTree: computing large minimum evolution trees with profiles instead of a distance matrix. Mol Biol Evol. 2009:26:1641-50.

63. Wang Q, Garrity GM, Tiedje JM, Cole JR. Naive Bayesian classifier for rapid assignment of rRNA sequences into the new bacterial taxonomy. Appl Environ Microbiol. 2007;73:5261-7.

64. Lozupone C, Hamady M, Knight R. UniFrac —an online tool for comparing microbial community diversity in a phylogenetic context. BMC Bioinformatics. 2006;7:371.

\section{Submit your next manuscript to BioMed Central and take full advantage of:}

- Convenient online submission

- Thorough peer review

- No space constraints or color figure charges

- Immediate publication on acceptance

- Inclusion in PubMed, CAS, Scopus and Google Scholar

- Research which is freely available for redistribution 